\section{OBSERVATIONS ON SEA-SICKNESS.}

\author{
By JAMES R. WORTABET, M.B. \\ Late Surgeon R.M.S. Dunvegan Castle, \\ Twickenham.
}

IN reference to the paper by Dr. C. Butler Savory on seasickness in the British Medical Journal of March 30 th, and as my experience of the malady differs from his, it may be of interest if I give an account of my observations. I have acted as a ship's surgeon for a considerable period of time. I have travelled more than 100,000 nautical miles, and I have had usually under my care several hundred passengers besides the crew. Though well inured to sea life I myself have suffered on one or two occasions, and I found I nearly always did so when 1 went to sea with loaded bowels, and presumably a full gall bladder.

My own feelings and observation led me to believe that all my symptoms were referable to the abdominal organs, none to the cerebral, although I quite believe in two distinct types of the sickness. I am quite convinced that certain precautionary methods adopted by experienced travellers almost completely prevent their being affected to any extentnamely, by taking a saline purgative the day or so before travelling, by adopting the recumbent position, and on all occasions avoiding oleaginous smells and the company of those who are sea-sick, as example seems a powerful excitant to sea-sickness.

There are also certain stomachic sedatives which, if taken at once, undoubtedly tend to prevent sickness, notably potassium bromide and some similar drugs; but, having given a fair trial to all the most lauded preparations which have been brought forward as panaceas, I have found none of them infallible. I have noticed that patients who went at once to sleep on coming aboard, either from the effects of a dose of chloral taken at their own instigation, or from the effects of alcohol, often escaped the malady, which on other occasions they did not. But to show how uncertain deductions from this statement are, I may mention that both sailors and firemen not infrequently come on board the worse for liquor, and almost invariably are sea-sick before getting into their normal condition.

I believe that more depends on the kind of motion of the ship than on nearly anything else, although, as I have pointed out, the recumbent position, renders one less liable to this ailment than the erect. With a head sea and slight pitching far more people are affected than even with severe rolling, and singularly the majority of the people affected, if asked to describe their sensations, refer them in nearly all cases to the abdomen primarily, and I can myself corroborate this experience, the downward motion of the vessel seems to have distinctly more effect than the upward. An experience I had with about 500 Hebrew pilgrims at Jaffa most strongly confirms the above statement. I do not at all question the statement that a certain proportion of cases have their origin in the cerebral centres primarily, but I should not attempt to venture on a distinct localisation. It has been pointed out, with what certainty I cannot tell, that the sensations occasionally experienced in a train passing through a tunnel or in a lift in motion are in some way referable to the superior semicircular canal. Dr. Savory ascribes the whole mischief to the disturbance of the endolymph; but if such be the case, why should the vast majority of people suffer considerably more, as I have shown above, by slight pitching than by much more severe rollingt? In the cerebral cases pallor, giddiness, obscured vision, and other sensations precede the gastric phenomena, but my experience goes to prove that such cases are in the minority.

New modes of treatment are always cropping up, and each has its strenuous advocates; at one time Chapman's icebags to the spine, at other hot-water bottles to the feet and epigastrium. For my own part I am certain that in severe retching and persistent sickness nothing is so trustworthy as a hypodermic injection of morphine. With this exception I never rely alone on one method of treatment, for even sea-sick patients have their idiosyncrasies.

The best advice I can give-in a few words-to those who suffer principally from gastric phenomena is that before starting on a voyage they should provide themselves with a good flannel roller bandage 12 feet in length and 6 inches in breadth, and wind it round their trunk over the whole width of the abdominal region; this will frequently afford great comfort by preventing the contents of the viscera from undue movement. A few turns of a surgical bandage round the head also appear to allay a good deal of the accompanying headache.

\section{MEMORANDA:}

\section{MEDICAL, SURGICAL, OBSTETRICAL, THERA- PEUTICAL, PATHOLOGICAL, ETC.}

URINE, UREA, AND FORMOL.

Mr. E. A. Cook's interesting observation on the action of formalin on acid urine has been known for some years. I have still in my possession a sample of urinary sediment four years old, which contains numbers of these crystalline spherules. I first noticed these bodies in a highly acid urine which contained oxalates and spermatozoa, after the addition of formalin to preserve the sediment. Sometimes the spherules posses: a nucleus which is colourable by staining solutions. I have observed the formation of these bodies in urine which con tained uric acid crystals, and in urine which deposited neither uric acid nor oxalates.

London, S.W.

R. G. HEBB.

\section{HAMATURIA FOLLOWING THE ADMINISTRATION OF} UROTROPINE.

HAVING read the report of two cases of hæmaturia caused by urotropine in the British Medical Journal of June 15 th, I wish to report a case of albuminuria from the same cause.

The patient was a servant, aged $2 \mathrm{I}$, and after a mild attack of typhoid fever was up and on tish diet. She was put on ro grs. of urotropine three times a day; next day she complainéd a little of urinary pain, which was not reported to the medical officer. On the tenth day of taking it she was quite ill, with severe backache, swelling of eyelids, and weakness. The urine on boiling became almost solid with albumen.

The urotropine was discontinued, and next day the albumen had almost and on the third day had quite disappeared, and the patient continued well.

A. Griffith, M.O. Hove Sanatorium.

RECENTLY I had a case of hæmaturia following on administration of urotropine. The patient, a girl of 18 , was put on urotropine, 5 grains three times a day, in order to product. diuresis. After continuing to take the urotropine for seven days patient says that she experienced an uncomfortable irritation in the bladder, accompanied by desire to pass water frequently. Following on this hæmaturia commenced, and according to her own statement, in considerable quantity. Not considering anything was specially wrong she continued to take the urotropine for two days, and then, as matters seemed to get worse, she came again for advice. On examining a specimen a considerable amount of blood was discovered. The urotropine was immediately discontinued, with the result that the hæmaturia ceased and the urine became normal in two days' time. It seemed to me at the time that the hæmaturia was, in all probability, due to the urotropine, a suspicion confirmed by Dr. Langdon Brown's note.

Stamford:Hill, $\mathrm{N}$.

W. A. Milligan.

ON May I 5 th I was called to see W. F., aged 62, whom 1 found to be suffering from symptoms of cystitis of about ten days' duration. The urine contained pus, but no blood, and was ammoniacal. There was enlargement of the prostate.

I prescribed ro grs. of urotropine thrice daily in half a pint of warm water. Two days later he expressed himself as feeling better, and the urine had improved. On the evening of May 18th I was sent for, and found him suffering from increased difficulty in micturition, a burning feeling in the urethra, abdominal pain with diarrhœa, and marked hæmaturia, which latter symptom had commenced in the after- 